\title{
Modulation of initial movement for double potential targets with specific time constraints
}

Ryoji Onagawa ( $\square$ ryoji.onagawa@gmail.com )

The University of Tokyo https://orcid.org/0000-0002-4722-1981

Kazutoshi Kudo

The University of Tokyo https://orcid.org/0000-0002-3199-9449

Article

Keywords:

Posted Date: April 26th, 2021

DOI: https://doi.org/10.21203/rs.3.rs-460918/v1

License: (9) (1) This work is licensed under a Creative Commons Attribution 4.0 International License. Read Full License 


\section{Abstract}

In goal-directed behavior, individuals are often required to plan and execute a movement with multiple competing reach targets simultaneously. The time constraint assigned to the target is an important factor that affect the initial movement planning, but the adjustments made to the starting behavior considering the time constraints specific to each target have not yet been clarified. The current study examined how humans adjusted their motor planning for double potential targets with independent time constraints under a go-before-you-know situation. The results revealed that the initial movements were modulated depending on the time constraints for potential targets. However, under tight time constraints, the performance in the double-target condition was lower than the single-target condition, which was a control condition implemented to estimate performance when one target is ignored. These results indicate that the initial movement for multiple potential targets with independent time constraints can be modified, but the planning is suboptimal.

\section{Introduction}

In goal-directed behaviors, individuals are often required to perform movements under tight time constraints. This is especially pertinent to many sports, where decisions are made under severe time constraints, e.g., a defender responding to an opponent's dribble in ball games such as soccer or basketball. In these situations, motor plans are selected considering multiple possibilities for motor goals; however, the action often needs to be initiated and continued before a single motor goal is defined. Several studies have investigated motor planning for multiple potential goals using the "Go-before-youknow paradigm," in which a participant is required to launch a movement with multiple potential goals and the final goal is revealed after movement development (i.e., movement onset or reaching a given threshold) ${ }^{1-10}$. In general, when there are multiple potential goals at movement onset, the initial movements were found to be directed toward the average direction of the potential targets $1-3,5,6,11,12$

The performance of a goal-directed movement is traditionally conceptualized in two discrete phases: the planning phase and the execution phases ${ }^{13}$. Based on this discrete view, several models have been created to solve the redundancy problem (for instance, when reaching for an object, there are numerous hand trajectories, joint movements, and muscle activation patterns that can be executed on a single target). To solve this problem, optimization based on various costs, such as jerks ${ }^{14}$, torque-changes ${ }^{15}$, and variabilities of the final hand position ${ }^{16}$, have been proposed, and these perspectives have contributed greatly to our understanding of human motor control principles. However, although these traditional serial models ${ }^{17}$ assume that we first select a goal and then specify and prepare the corresponding goal-directed movement, selection and specification can operate as a continuous and parallel processes ${ }^{18,19}$. For example, the behavioral findings from recent studies have revealed that the movement trajectories in the simultaneous presence of multiple potential targets are directed in directions where no target exists ${ }^{1}$. Furthermore, human and primate neurophysiology studies have shown that 
competing reach targets induce separate neural representations corresponding to each target in sensorymotor brain areas before one of the targets is selected ${ }^{20-23}$.

Many behavioral studies using go-before-you-know tasks (i.e., tasks in which participants are presented with a large number of potential reaching targets simultaneously before knowing the final target location and are required to initiate a reaching movement to a competing target) have confirmed that humans initiate reaching movements toward the average of the potential targets $10,12,24$. However, such averaging behavior is less likely to be selected when the advantage of the strategy disappears, such as when the distance between targets is large ${ }^{7}$, when severe constraints on speed are imposed ${ }^{8}$, or when the information on the targets is updated in stages ${ }^{25}$. These findings suggest that the averaging behavior in motor planning in the presence of multiple goals may not exist as a control policy in itself, but is rather a behavior that reflects optimization for task accomplishment.

In a go-before-you-know-paradigm, the effect of time constraints assigned to potential targets on motor planning has not been sufficiently investigated. The time constraint is a critical factor because the motor target is often time-constrained, and the time constraint affects the possible movement dynamics. In addition, the effect of asymmetry between potential targets on motor planning has not yet been discussed. In many situations, different motor targets have specific time constraints, and "how do humans execute movements in situations where targets with different time constraints exist simultaneously?" seems to be an essential question. Therefore, in the current study, we investigated how to adjust the initial movements according to the time constraints assigned to each potential target.

The time constraint is an effective experimental control variable for examining how asymmetry in potential targets is reflected in motor planning. Although this approach of experimentally manipulating time constraints is similar to that used by Wong \& Haith $(2017)^{8}$, who restricted the movement velocity, or that used by Hesse et al. (2020) ${ }^{26}$, who manipulated the positioning of the target to manipulate the optimality of the averaging strategy, two reasons exist as to why manipulating time constraints may be effective for further understanding of behaviors. First, unlike constraints on movement speed, time constraints do not directly constrain the initial movement itself. Since the participants themselves are free to choose the initial movement, manipulation of the time constraint would be more appropriate for examining the adaptability of the motor planning to the given target information. Next, specific time constraints can be assigned to each target when manipulating the time constraint, thus creating an asymmetry in the temporal values of potential targets. Such manipulation of the time constraint can be used to examine how humans plan their movements to account for asymmetric temporal values of potential targets, while this approach is difficult to employ in studies on movement velocity.

Although motor planning for potential targets has been suggested to reflect optimization of success probability ${ }^{8}$, the extent of this optimality has not been clarified. The direction and velocity of the initial movement, as well as the corrective actions after the movement, are considered to reflect the strategy selected in advance by the participants. In this case, an important decision about the strategy for two potential targets is whether to focus on one target (i.e., a predetermined strategy) or to take both targets 
into account (i.e., a choice-reaction strategy). Previous studies confirmed that humans prefer a choicereaction strategy even in situations with severe spatiotemporal constraints to sufficiently accomplish a task ${ }^{26,27}$. A similar bias may exist in the selection of strategies when encountering asymmetric time constraints in the go-before-you-know task. Thus, the present study examined whether pre-determined or choice-reaction strategies can be optimally selected to maximize the success rate under extremely tight time constraints by comparing the performance when two targets are present with the performance when only one target is present.

Therefore, the current study used a go-before-you-know task with two targets to examine how a combination of the time constraints assigned to each target is considered in motor planning. Participants started the movement with the time constraints of each target known in advance, and the final target was specified after the movement onset. Participants were considered successful when they reached the final target within the time constraint. The time constraint for each target was randomly assigned for each trial in the range of $200-1000 \mathrm{~ms}$. Participants were required to maximize the success probability within the set (50 trials). As a control condition, participants also performed trials with only one target before the start of the movement. The kinematic properties (i.e., direction and velocity) and optimality of the planning of the initial movement were tested by examining the variations in the direction and velocity of the initial movement in the double-target condition depending on the time constraints, and by comparing the initial movement and performance in relation to the number of potential targets. More specifically, we first examined the changes in motor patterns related to combinations of time constraints, based on movement trajectories and the bivariable histograms of and kinematic properties of the initial movement. We also categorized the patterns of initiating actions in a data-driven manner using k-means clustering and examined how the ratio of occurrences of each pattern changes depending on the combination of time constraints. In addition, we examined the variation in the initial movement pattern and performance depending on the time constraint and number of targets.

\section{Results}

\section{Modulation of the initial movement according to the combination of time constraints}

First, we descriptively examined the changes in the initial movement according to the combination of time constraints. Figure 2 shows the trajectories of cursors classified by time constraints, including the data of all participants (the trajectories of each participant are shown in the supplementary information). This figure suggests that the selected trajectory modulates according to the time constraint. From a qualitative perspective, the initial movement was divided into three main directions: center direction, left target direction, and right target direction. While movement in the center direction was observed within any time constraint, movement in the target directions was mainly observed in the direction with tighter time constraints. Modulation dependent on the time constraint was also clearly seen in the bivariate histograms of the initial movement direction (IMD) and initial movement velocity (IMV), as shown in Fig. 3. More specifically, when the time constraints were comparable, a high frequency of initial movement in the center direction and a symmetrical distribution along $90^{\circ}$ of the IMD were confirmed. On 
the other hand, under conditions where the difference in time constraints was large (lower left or upper right panel), the frequency of the initial movement in the target direction with tighter time constraints was high, although initial movement in the center direction also existed.

To quantitatively demonstrate the qualitative observations highlighted in Fig. 3, the initial movement patterns (IMD and IMV) were classified into three clusters by using k-means clustering, and the percentage of occurrence of each pattern was compared. This analysis could more clearly quantify the modulation of initial movement patterns, although comparison of average values is associated with the problem that the values were equivalent when the movement was directed to either the left or right target with the same frequency, or when the movement was directed at the center. Figure 4 shows the scatter plots of the IMD and IMV, including all data for all participants, and the occurrence probability of each cluster for each combination of time constraints. This result quantitatively illustrates the qualitative observations shown in Fig. 3. The scatter plots indicated that the initial movement in the center direction was relatively slow, and the initial movement in the target direction was relatively fast. This difference in velocity may reflect differences in the subsequent correction strategy. A three-way repeated-measures ANOVA (3 [cluster] $\times 3$ [left time constraint] $\times 3$ [right time constraint]) on the occurrence probability revealed a three-way interaction $\left(F_{[8,88]}=2.626, \eta^{2}{ }_{p}=0.193, p=.013\right)$. Post-hoc one-way ANOVAs (3 [cluster]) and multiple comparisons in each combination of time constraints are shown in Table 1, which reveals that the occurrence probability of each cluster depending on time constraints suggested that the proportion of movement directions (i.e., left, center, or right) varied depending on the combination of time constraints assigned to each target. In the condition where the time constraint did not differ between potential targets, the middle direction was selected more frequently, and when the time constraint differed, the target direction with a shorter time constraint was selected more frequently. These results showed that participants selected different initial movements depending on the time constraint.

\section{Comparison of initial movement and performance between the double-target and single-target conditions}

Figure 5 shows an inter-condition comparison of the IMD, IMV, and | $\triangle I M D \mid$ between the double-target and single-target conditions. $|\triangle I M D|$ was defined as the absolute value of the angle difference between the IMD and the vertical vector, and it evaluated whether the initial movement was directed in the target direction or the center direction. A two-way repeated-measures ANOVA (5 [time constraints on left target] $\times$ 5 [time constraints on right target]) of the IMV revealed significant main effects of time constraints on the left target $\left(F_{[4,44]}=22.456, \eta^{2}{ }_{p}=0.671, p<.001\right)$ and time constraints on the right target $\left(F_{[4,44]}=15.436\right.$, $\left.\eta_{p}^{2}=0.584, p<.001\right)$ and no significant interaction $\left(F_{[16,176]}=1.363, \eta_{p}^{2}=0.11, p=.165\right)$. However, a twoway repeated-measures ANOVA (5 [time constraints on left target] $\times 5$ [time constraints on right target]) of the IMD revealed significant main effects of time constraints on the left target $\left(F_{[4,44]}=23.296, \eta^{2} \mathrm{p}=\right.$ $0.679, p<.001)$ and time constraints on the right target $\left(F_{[4,44]}=7.697, \eta^{2}{ }_{p}=0.412, p<.001\right)$ and a significant interaction $\left(F_{[16,176]}=3.669, \eta_{p}^{2}=0.25, p<.001\right)$. Similarly, a two-way repeated-measures ANOVA (5 [time constraints on left target] $\times 5$ [time constraints on right target]) of the $|\triangle I M D|$ revealed significant main effects of time constraints on the left target $\left(F_{[4,44]}=8.114, \eta^{2} p=0.425, p<.001\right)$ and time constraints on the right target $\left(F_{[4,44]}=10.154, \eta_{p}^{2}=0.48, p<.001\right)$ and a significant interaction 
$\left(F_{[16,176]}=2.437, \eta_{p}^{2}=0.181, p=.002\right)$. These results suggest that the velocity varies according to the time constraints of both targets. Especially in the initial movement angle, the movement was initiated toward the target with a shorter time constraint (as in Fig. 2-4), but more importantly, it deviated more toward the center than the single-target condition. This central tendency seemed to reflect the fact that instead of employing a predetermined strategy, the participants had chosen an initial movement that was intended to reach both potential targets.

Figure 6 shows an inter-condition comparison of the temporal performance, arrival direction accuracy, and overall performance. For the arrival direction accuracy and overall performance, the black color indicates the data of the single-target condition multiplied by $1 / 2$ to estimate the performance when the predetermined strategy is adopted. The temporal performance in the double-target condition was confirmed to be lower than that in the single-target condition. Moreover, the arrival direction accuracy in the double-target condition was relatively higher than that in the single-target condition (50\% accuracy), although it was slightly lower in the condition with severe time constraints. This indicated that the choicereaction strategy was used more frequently than the predetermination strategy, and that even though the initial movement was directed in either target direction, the movement was eventually carried out in the direction of the final target.

Because the performance of the double-target condition seemed to be lower than that of the single-target condition under the tightest time constraint, an exploratory analysis was conducted to examine the effect of the other time constraint condition and the number of targets under the condition where one of the time constraints was the most severe. A two-way repeated-measures ANOVA (2[number of targets] $\times$ 5 [time constraints]) on the overall performance revealed significant main effects of both the number of targets (left fixed: $F_{[16,176]}=28.132, \eta_{p}^{2}=0.719, p<.001$, right fixed: $F_{[16,176]}=16.628, \eta_{p}^{2}=0.602, p$ $=.002$ ) and time constraints (left time-constraint fixed: $F_{[16,176]}=5.877, \eta_{p}^{2}=0.348, p<.001$; right timeconstraint fixed: $\left.F_{[16,176]}=6.019, \eta_{p}^{2}=0.354, p<.001\right)$. These results suggest that the overall performance in the double-target condition seemed to be higher than that of the predetermined strategy when the time constraint was relatively loose, but the predetermined strategy performed better when the time constraint was severe (especially when the red-colored data were checked). These results indicate that even in situations where time constraints are tight and a predetermined strategy is desirable, a selective strategy is adopted, resulting in poor performance.

\section{Discussion}

The current study examined how an initial movement is selected for a given time constraint for each potential target and whether the selection of the strategy for a given time constraint is desirable in terms of success probability. The results showed that the initial movement evaluated by the motor variables $100 \mathrm{~ms}$ after the movement onset varied according to the given time constraint, suggesting that participants adjust their behavior depending on the combination of time constraints. Specifically, in situations where the severities of the time constraints were comparable, the initial movement was directed toward the average direction of the potential targets, and in situations where there was a 
substantial difference in the severities of the time constraints, the initial movement was directed toward the target direction with a more severe time constraint. In addition, a comparison of the initial movement and performance between the double-target and single-target conditions revealed that a selective strategy was adopted even when a predetermined strategy was desirable.

Previous studies have reported that in the presence of multiple potential targets, humans vary the initial movement depending on the condition of motor planning. For instance, although the participants selected an initial movement in an intermediate direction at a relatively slow movement speed, such an initial movement was no longer selected, and the initial movement headed linearly to one of the potential targets at the fast movement speed required ${ }^{8}$. These results indicate that the planning of the initial movement under multiple potential targets reflects the optimization of the task. A recent study reported that motor planning (in particular, preparation of feedback responses) is adjusted in a utility-dependent manner when asymmetry exists in the utility assigned to the target ${ }^{28}$. These studies showed that motor planning may purposefully depend on restrictions of motor execution (e.g., speed and direction) and target information (e.g., value and size).

As an extension of the above study, the current study experimentally manipulated the time constraints assigned to each potential target and investigated the modulation of the initial movement according to the combinations of time constraints. The results showed that the velocity and direction of the initial movement were modulated according to the combination of time constraints. Specifically, the participants generally initiated their movements in the middle direction of the potential target in conditions with equal time constraints. In the conditions where the time constraints differed, participants generally initiated their movements in the direction of the target with the shorter time constraint and corrected their movements when the target with the longer time constraint was correct. This strategy of initially prioritizing the target with a tighter time constraint and correcting the movement if the other target was the true target is considered to be a behavior that reflects the task demands: targets with short time constraints need to be reached quickly, while more time can be taken for targets with longer time constraints.

One possible explanation for the modulation of initiation behavior to multiple potential targets is task optimization or reward maximization 7,8 . However, the extent of optimality in such tasks has not yet been tested. In particular, an important aspect of strategy selection in a time-urgent situation is the decision to use a predetermined strategy or a choice-reaction strategy. In this case, optimality lies not in the ability to choose the more desirable strategy under certain extreme conditions (e.g., conditions where time constraints are too tight or too loose), but in the ability to switch strategies appropriately in situations where the expected outcomes are equivalent. The present study exploratively investigated whether switching between the predetermined and the choice-reaction strategy was appropriate by comparing the initiating behavior and performance between the single-target and double-target conditions. The results revealed that the temporal performance in the double-target condition was lower than that in the predetermined strategy (i.e., estimated by the behaviors in the single-target condition), and the probability of reaching the correct target direction was higher than that in the predetermined strategy, resulting in 
poorer outcomes when the time constraint was severe. Additionally, the direction of the initiating action was not always directed toward one target, although the probability of being directed toward the central direction decreased in the condition with severe time constraints. These results suggest that even in conditions where a predetermined strategy is desirable, there is a bias toward the choice-reaction strategy that results in poor performance.

We propose several possible causes for this preference bias in the choice-reaction strategy. First, inaccurate cognition of the sensorimotor system may lead to limited optimality. The normative perspective in the current study assumes that the decision-maker is aware of the exact relationship between the strategy and the outcome of the movement. However, it is unclear whether the participants actually recognized this relationship completely and accurately. Indeed, in a timing-coincidence task, the recognized outcome of one's own action was reported to be perceived closer to the success direction than the actual one ${ }^{29}$. Moreover, motor variance also tended to be more under-recognized than it actually is 30,31 , and there is consistency among individuals in the pattern of deviations from the optimal solution. This may be due to the biased recognition of one's own sensorimotor system.

Second, it is conceivable that participants found more value in spatial accuracy than in temporal accuracy. In the current task, the final outcome (obtaining a score) was determined by two dimensions: space and time. In tasks involving multidimensional elements in the final outcome, interpretation of the outcome of each dimension in an integrated manner remains a challenge. If participants try to reach both potential targets, they can reach the direction of the correct target even if they fail to reach it in time. In contrast, with a predetermined strategy, the participants will go in a different direction from the correct target in approximately half of the trials. With the choice-reaction strategy, even if they could not reach the target in time, they may find value in the fact that they were able to eventually reach the target location. Correspondingly, even if they had made it in time, they may have found a loss in reaching a direction where the target did not exist. Future research is necessary to understand how each factor is recognized and integrated when both time and space affect success or failure, as in the current task.

Another possibility is that the subjective utility of the strategy led to a systematic deviation from the better strategy. In a predetermined strategy, the outcome of the movement depends on external factors. As we underestimate the value of a "lucky hit," we may underestimate the value of a strategy that relies on such randomness. This may reflect an unwillingness to devote effort to action if there is a high likelihood that it will be futile or reflect a low estimate of the value of the action if it succeeds or a high estimate of the loss if it fails. If such a cognitive bias is at work, it could be a strong impediment to strategy optimization in situations with insufficient time to execute the targeted action. Importantly, the same tendency has been consistently observed in our previous studies ${ }^{27}$ and in other recent studies ${ }^{26,32}$, necessitating the identification of its causes.

Finally, in the current optimization norms, it is assumed that all possible motor plans are simulated, but all motor plans may not necessarily be simulated in a finite amount of time. One obvious possibility is that the participants' motor plans reflect an optimization of the entire task, rather than the optimization of 
only a given condition. In fact, in the range of conditions in the present experiment, the more desirable condition was the one in which the participants were trying to reach the two targets. Therefore, a choicereaction strategy may be adopted even in a minority of conditions where a predeterminant strategy is desirable. It is also known that humans tend to overestimate the loss caused by choosing an option that is not currently implemented and prefer to maintain the status quo ${ }^{33}$. Our daily strategy selections are made in the face of constantly changing circumstances. In such situations, we may not be optimizing locally for a given condition, but rather globally, including a wider range of time scales. Future studies are required to clarify the type of optimization performed when successively dealing with various situations.

\section{Methods}

\section{Participants}

Twelve right-handed neurologically healthy participants (age: $22.7 \pm 3.1$ years, ten men) were recruited. All patients had normal or corrected-to-normal vision, were naive to the objectives of this study, and provided written informed consent. This study was approved by the Ethics Committee of the Graduate School of Arts and Sciences, University of Tokyo. All experimental procedures adhered to approved guidelines for experimental procedures. Informed consent was obtained from each participant before the experiments in a written format.

\section{Experimental setup}

The participants sat in a quiet, dim room. A pen tablet with sufficient workspace to measure the subjects' arm reach movement (Wacom, Intuos 4 Extra Large; workspace: $488 \times 305 \mathrm{~mm}$ ) was set on the table. A monitor (I-O DATA, KH2500V-ZX2; 24.5 inches, $1920 \times 1080$ pixels, vertical refresh rate, $240 \mathrm{~Hz}$ ) was set for stimulus presentation with an approximately $30^{\circ}$ gradient angle over the pen-tablet. The participants manipulated a cursor on a screen whose position was transformed from the position of the pen. The time elapsed from the movement onset and the location of the cursor on the monitor were sampled at $240 \mathrm{~Hz}$. All stimuli were controlled using the Psychophysics Toolbox of MATLAB (MathWorks, Natick, MA, USA).

\section{Experimental task}

The participants performed a task-modified version of a go-before-you-know paradigm, in which two potential targets were presented on the screen and one of the targets was revealed as the final target only after the participant launched his/her movements. These targets appeared $20 \mathrm{~cm}$ away from the start position and were presented on either side of the midline at $+30^{\circ}$ and $-30^{\circ}$. Independent time constraints were randomly assigned to each target in each trial. The range of time constraints was 200 to $1000 \mathrm{~ms}$. The direction of the true target $\left(+30^{\circ}\right.$ or $\left.-30^{\circ}\right)$ was equally randomized in each condition and set. Each set included 50 trials.

Figure 1 shows the sequence of the trial. The sequence of tasks was almost the same for the doubletarget and single-target conditions. To begin the trial, participants moved a cursor (white frame oval, radius: $0.5 \mathrm{~cm}$ ) to a start position (white frame oval, radius: $0.5 \mathrm{~cm}$ ) presented on a screen (Fig. 1a). 
When the cursor reached the start position, it turned into a blue oval. Subsequently, the time bar indicating the time constraints assigned for each target was presented above the potential target position (Fig. 1b). After $1000 \mathrm{~ms}$, the potential targets are presented in Fig. 1c. After a random interval (450-1000 ms), an auditory beep cued the participant to initiate a movement. Participants were required to initiate a movement up to $500 \mathrm{~ms}$ after the beep cue. When the cursor was $1 \mathrm{~cm}$ away from the start position, the true target changed to a filled yellow circle, and the other target disappeared (Fig. 1d and Fig. 1e). After movement onset, the area of the gray bar decreased over time. When the cursor moved through the true target within a given time constraint for the target, the participants acquired 100 points. After the stimuli disappeared (1000 ms after movement onset, Fig. 1f), the results of the movements ("Hit" or "Miss") and the scores ( 0 points for a missed trial or 100 points for a successful trial) were presented as feedback (Fig. 1g). If movement onset time was more than 500 ms or less than 0 ms (i.e., movement onset was faster than a beep cue), "Too late" or "Too early" was presented on the screen as feedback, respectively. In this case, the participants acquired no points. The participants were instructed to maximize the average score for each set.

First, the double-target condition was performed for six sets of 50 trials. Then, the single-target condition was performed for two sets of 50 trials. The time constraint for each target was randomly assigned in the range of 200-1000 ms for each trial. Sufficient rest was taken between the sets to avoid fatigue.

\section{Data analysis}

The observed data were analyzed using programs written in MATLAB software (MathWorks, Natick, MA, USA). The data obtained in the last four sets in the double-target trials (200 trials) and all sets in the single-target trials (100 trials) were used for analysis. The cursor positions (horizontal position: $X c(t)$, vertical position: $Y c(t)$ ) at each time point $(t)$ were calibrated using a secondorder, zero-phase-lag, low-pass Butterworth filter with a cutoff frequency of 6 $\mathrm{Hz}$. Movement onset time was identified using a distance $0.5 \mathrm{~cm}$ away from the start position.

The movement onset time was identified using the cursor distance $(d(t)=$ $\left.\sqrt{x_{c}(t)^{2}+y_{c}(t)^{2}}\right) 5 \%$ of the target distance from the start position $(0.5 \mathrm{~cm})$. Reach time was defined as the time when the cursor distance $d(t)$ was longer than the distance from the start position to the target (i.e., $20 \mathrm{~cm}$ ). The cursor angle at each time point $(\mathrm{t})$ was determined as the angle between the vector from the start position to the cursor position and the horizontal vector. The IMD was determined as the cursor angle at $100 \mathrm{~ms}$ after the movement onset, while the IMV was determined as the movement velocity $100 \mathrm{~ms}$ after movement onset.

\section{Clustering of the initial movement}


To classify the characteristics of the initial movement, k-means clustering was used to classify the initial movement into three clusters based on the combination of the initial movement direction (IMD) and initial movement velocity (IMV).

\section{Comparison of initial movement and performance between the double-target and single-target conditions}

The combinations of time constraints for the left and right targets were classified as $5 \times 5$, and the means of IMV, IMD, and $|\triangle I M D|$ were calculated for each. $|\triangle I M D|$ is defined as the absolute value of the angle difference between the IMD and vertical vector. In addition, within the same classification of time constraints, the means of temporal performance, arrival direction accuracy, and overall performance were calculated for each individual. Temporal performance denotes the probability that the reach time is less than the time constraint, regardless of whether the target was hit or not. The arrival direction accuracy indicates the probability that the arrival direction matches the correct target and does not consider whether the target can be hit correctly. Overall performance indicates the probability of success (i.e., whether the hit is accurate, in time). These indices were compared between double-target and singletarget conditions.

\section{Statistical analysis}

To test the modulation of the initial movement depending on the time constraints, three-way repeatedmeasures ANOVAs (3 [cluster] $\times 3$ [left time constraint] $\times 3$ [right time constraint]) of the occurrence probability and two-way repeated-measures ANOVAs (5 [time constraints on left target] $\times 5$ [time constraints on right target] of the IMV, IMD, and $|\triangle I M V|$, and post-hoc tests were conducted. In addition, under the condition where one of the time constraints was the most severe, the effect of the other time constraint condition and the number of targets, two-way repeated-measures ANOVAs (2[number of targets] $\times 5$ [time constraints]) of the overall performance were conducted with either the left or right timeconstraint fixed.

\section{Declarations}

\section{Acknowledgments}

The research was supported by Grant-Aid for JSPS Fellows No. 18J23290 awarded to R.O. and by JSPS KAKENHI No. 26560344 and $20 \mathrm{H} 04069$ awarded to K.K.

\section{Author contributions}

R.O. and K.K. designed the experiments. R.O. performed the experiments. R.O. programmed and analyzed the results. R.O. and K.K. interpreted the results. R.O. wrote the paper under the supervision of K.K.

\section{Competing interests}

The authors declare no competing interests. 


\section{References}

1. Chapman, C. S. et al. Reaching for the unknown: Multiple target encoding and real-time decisionmaking in a rapid reach task. Cognition 116, 168-176 (2010).

2. Stewart, B. M., Baugh, L. A., Gallivan, J. P. \& Flanagan, J. R. Simultaneous encoding of the direction and orientation of potential targets during reach planning: evidence of multiple competing reach plans. J. Neurophysiol. 110, 807-816 (2013).

3. Stewart, B. M., Gallivan, J. P., Baugh, L. A. \& Flanagan, J. R. Motor, not visual, encoding of potential reach targets. Curr. Biol. 24, R953-R954 (2014).

4. Hudson, T. E., Maloney, L. T. \& Landy, M. S. Movement planning with probabilistic target information. J. Neurophysiol. 98, 3034-3046 (2007).

5. Gallivan, J. P., Barton, K. S., Chapman, C. S., Wolpert, D. M. \& Randall Flanagan, J. Action plan cooptimization reveals the parallel encoding of competing reach movements. Nat. Commun. 6, 7428 (2015).

6. Gallivan, J. P., Bowman, N. A. R., Chapman, C. S., Wolpert, D. M. \& Flanagan, J. R. The sequential encoding of competing action goals involves dynamic restructuring of motor plans in working memory. J. Neurophysiol. 115, 3113-3122 (2016).

7. Haith, A. M., Huberdeau, D. M. \& Krakauer, J. W. Hedging your bets: Intermediate movements as optimal behavior in the context of an incomplete decision. PLoS Comput. Biol. 11, 1-21 (2015).

8. Wong, A. L. \& Haith, A. M. Motor planning flexibly optimizes performance under uncertainty about task goals. Nat. Commun. 8, 14624 (2017).

9. Nashed, J. Y., Diamond, J. S., Gallivan, J. P., Wolpert, D. M. \& Flanagan, J. R. Grip force when reaching with target uncertainty provides evidence for motor optimization over averaging. Sci. Rep. 7, 1-8 (2017).

10. Krüger, M. \& Hermsdörfer, J. Target uncertainty during motor decision-making: The time course of movement variability reveals the effect of different sources of uncertainty on the control of reaching movements. Front. Psychol. 10, 1-13 (2019).

11. Ghez, C. et al. Discrete and continuous planning of hand movements and isometric force trajectories. Exp. brain Res. 115, 217-33 (1997).

12. Gallivan, J. P. et al. One to Four, and Nothing More. Psychol. Sci. 22, 803-811 (2011).

13. Woodworth, R. S. Accuracy of voluntary movement. Psychol. Rev. Monogr. Suppl. 3, i-114 (1899).

14. Flash, T. \& Hogan, N. The coordination of arm movements: an experimentally confirmed mathematical model. J. Neurosci. 5, 1688-703 (1985).

15. Uno, Y., Kawato, M. \& Suzuki, R. Formation and control of optimal trajectory in human multijoint arm movement. Biol. Cybern. 61, 139-47 (1989).

16. Harris, C. M. \& Wolpert, D. M. Signal-dependent noise determines motor planning. Nature 394, 780784 (1998). 
17. McClelland, J. L. On the time relations of mental processes: An examination of systems of processes in cascade. Psychol. Rev. 86, 287-330 (1979).

18. Cisek, P. \& Centre-ville, C. P. S. Cortical mechanisms of action selection: the affordance competition hypothesis. 1585-1599 (2007). doi:10.1098/rstb.2007.2054

19. Gallivan, J. P., Chapman, C. S., Wolpert, D. M. \& Flanagan, J. R. Decision-making in sensorimotor control. Nat. Rev. Neurosci. 19, 519-534 (2018).

20. Cisek, P. \& Kalaska, J. F. Neural correlates of reaching decisions in dorsal premotor cortex: Specification of multiple direction choices and final selection of action. Neuron 45, 801-814 (2005).

21. Coallier, É., Michelet, T. \& Kalaska, J. F. Dorsal premotor cortex: Neural correlates of reach target decisions based on a color-location matching rule and conflicting sensory evidence. J. Neurophysiol. 113, 3543-3573 (2015).

22. Cui, H. \& Andersen, R. A. Different Representations of Potential and Selected Motor Plans by Distinct Parietal Areas. J. Neurosci. 31, 18130-18136 (2011).

23. Dekleva, B. M., Ramkumar, P., Wanda, P. A., Kording, K. P. \& Miller, L. E. Uncertainty leads to persistent effects on reach representations in dorsal premotor cortex. Elife 5, 1-24 (2016).

24. Chapman, C. S. et al. Reaching for the unknown: Multiple target encoding and real-time decisionmaking in a rapid reach task. Cognition 116, 168-176 (2010).

25. Onagawa, R. \& Kudo, K. Flexible planning of corrective responses for double - step reduction in the number of potential targets. Sci. Rep. 1-11 (2021). doi:10.1038/s41598-021-86325-9

26. Hesse, C., Kangur, K. \& Hunt, A. R. Decision making in slow and rapid reaching: Sacrificing success to minimize effort. Cognition 205, 104426 (2020).

27. Onagawa, R., Shinya, M., Ota, K. \& Kudo, K. Risk aversion in the adjustment of speed-accuracy tradeoff depending on time constraints. Sci. Rep. 9, (2019).

28. Carroll, T. J., Mcnamee, D., Ingram, J. N. \& Wolpert, D. M. Rapid visuomotor responses reflect valuebased decisions Rapid visuomotor responses reflect value-based decisions 2 Abbreviated title: Visuomotor reflexes reflect value-based decisions 3. Cite as J. Neurosci (2019). doi:10.1523/JNEUROSCI.1934-18.2019

29. Wolpe, N., Wolpert, D. M. \& Rowe, J. B. Seeing what you want to see: Priors for one's own actions represent exaggerated expectations of success. Front. Behav. Neurosci. 8, 1-14 (2014).

30. Zhang, H., Daw, N. D. \& Maloney, L. T. Testing Whether Humans Have an Accurate Model of Their Own Motor Uncertainty in a Speeded Reaching Task. PLoS Comput. Biol. 9, (2013).

31. Yamamoto, H., Shinya, M. \& Kudo, K. Cognitive Bias for the Distribution of Ball Landing Positions in Amateur Tennis Players ( Cognitive Bias for the Motor Variance in Tennis ) Cognitive Bias for the Distribution of Ball Landing Positions. 2895, (2018).

32. Clarke, A. D. F. \& Hunt, A. R. Failure of Intuition When Choosing Whether to Invest in a Single Goal or Split Resources Between Two Goals. Psychol. Sci. 27, 64-74 (2016). 
33. Kahneman, D., Knetsch, J. L. \& Thaler, R. H. Anomalies: The Endowment Effect, Loss Aversion, and Status Quo Bias. in Choices, Values, and Frames 159-170 (Cambridge University Press, 2000). doi:10.1017/CBO9780511803475.009

\section{Figures}

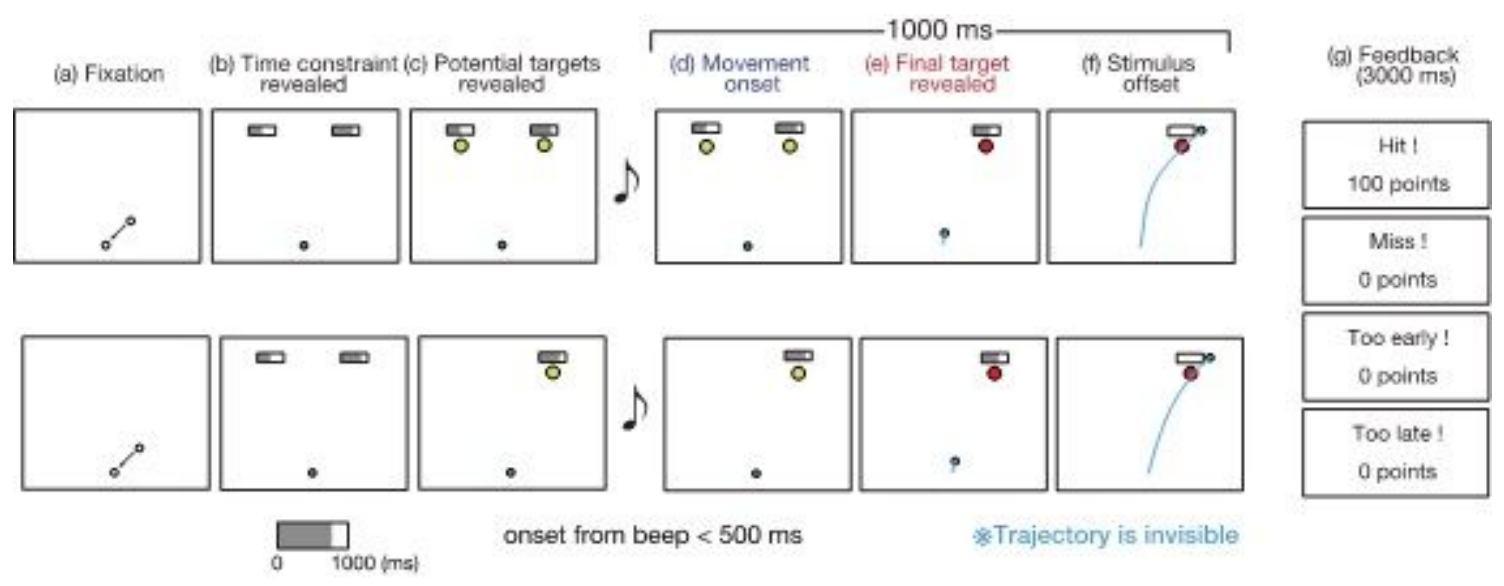

Figure 1

Sequence of the experimental task. The top row shows the double-target condition. The bottom row shows the single-target condition. (a) First, participants move the cursor to the start position. (b) After the presentation of the time constraint indicator, (c) the potential targets are presented. (d) 450-1000 ms later, participants were required to start the movement after the sound stimulus. After the movement onset, the gray area of the indicator reduces linearly with time. (e) When the participant's movement onset is detected, the final target is presented. (f) The stimulus disappeared $1000 \mathrm{~ms}$ after the movement onset. Participants acquired 100 points if they met the movement onset criteria and passed the final target within the time constraint assigned to the final target. (g) Feedback on successes, failures, and scores was provided after each movement. 
Time constraint (right target)

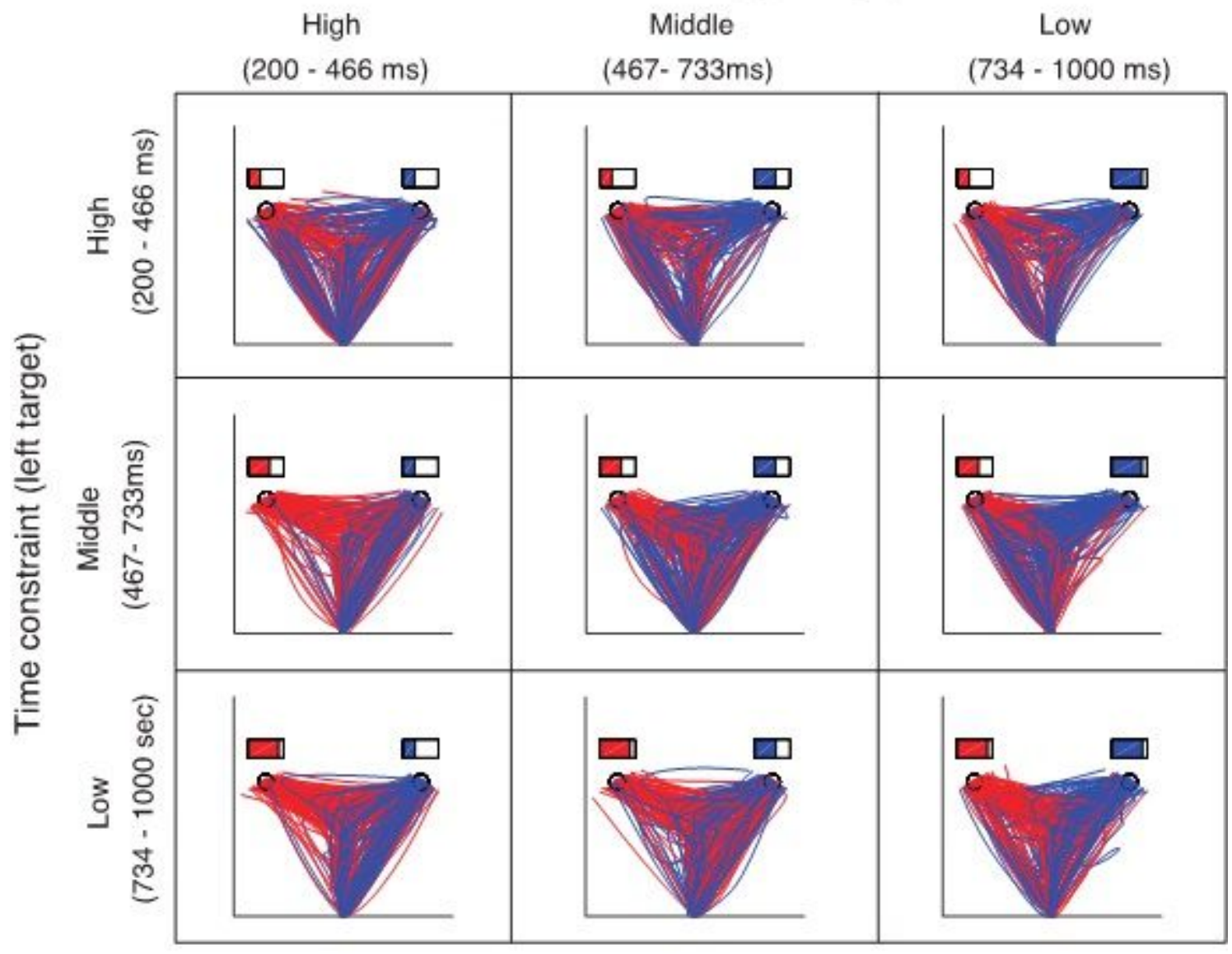

(All particiapant's data)

\section{Figure 2}

Movement trajectories according to combinations of the time constraints. The movement trajectories according to the time constraint of all participants in the double-target condition are shown. The color is based on the final target (red: final target is left, blue: final target is right). This figure confirmed that the trajectories of the participants' movements are modulated according to the combination of time constraints. For combinations of equivalent time constraints, the trajectories seemed to be bilaterally symmetrical. On the other hand, in situations with different time constraints, the frequency of the initial movement to the target with shorter time constraints seemed to be higher. 


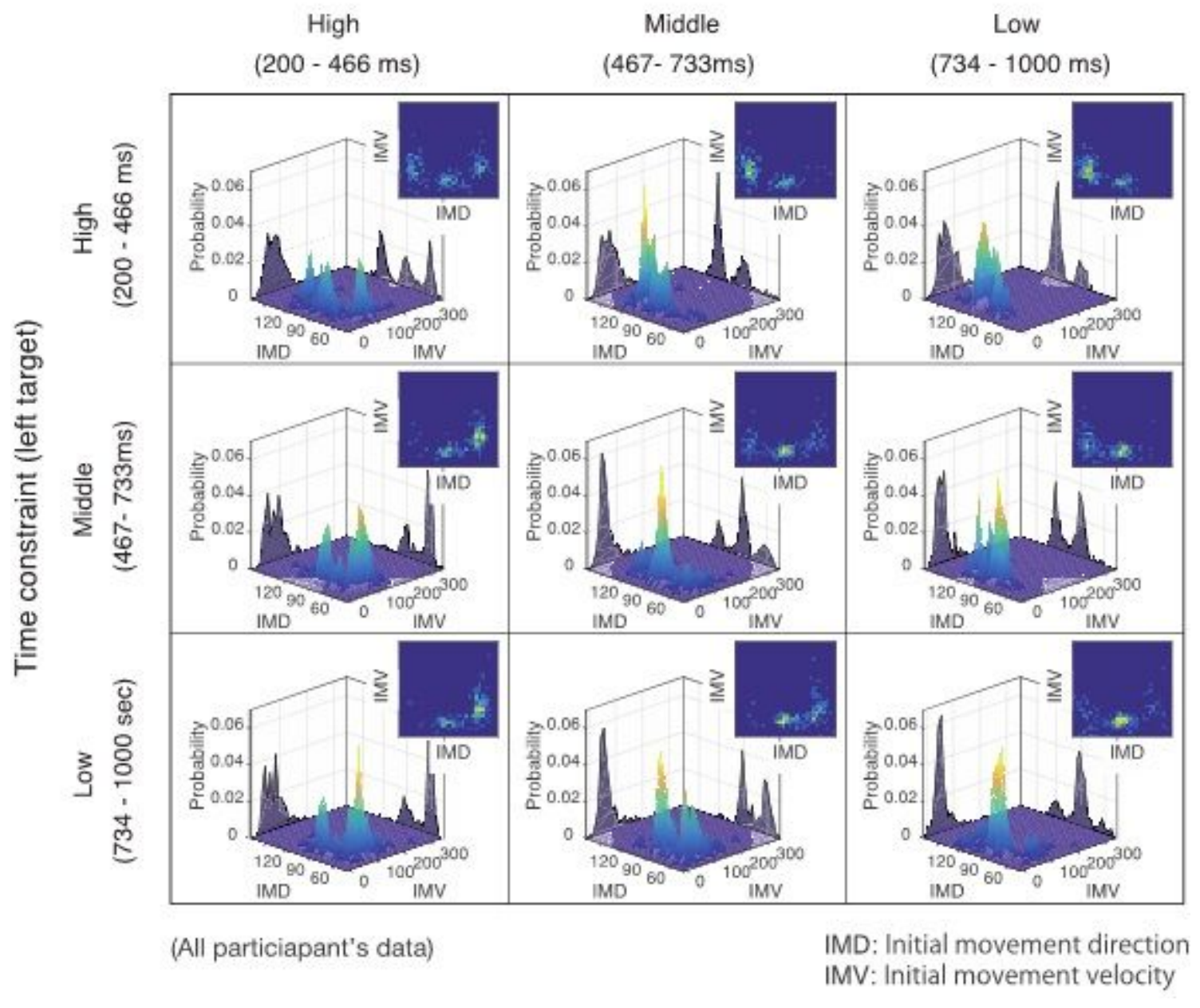

Figure 3

Bivariate histograms of the initial movement direction (IMD) and the initial movement velocity (IMV) according to the combinations of the time constraints. The bivariate histograms of the IMD and the IMV according to the time constraint in the double-target condition are shown. The center of each box shows the bivariate histogram, and the upper right corner shows the same histogram viewed from the vertical direction. As in Fig 2, this Figure shows the change in the direction and velocity of the initial movement depending on the time constraint. In particular, the diagonal line from the upper left to the lower right shows a high frequency of initial movement in the center direction and a symmetrical distribution along $90^{\circ}$ of the IMD. On the other hand, in the conditions where the difference in time constraints is large (lower left or upper right panel), the frequency of the initial movement in the target direction with tighter time constraints is high, although initial movement in the center direction also exists. 

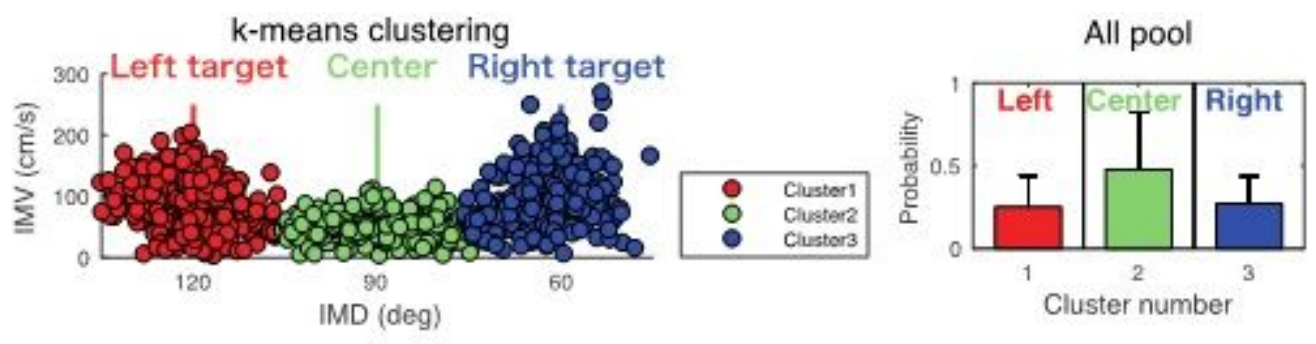

Time constraint (right target)

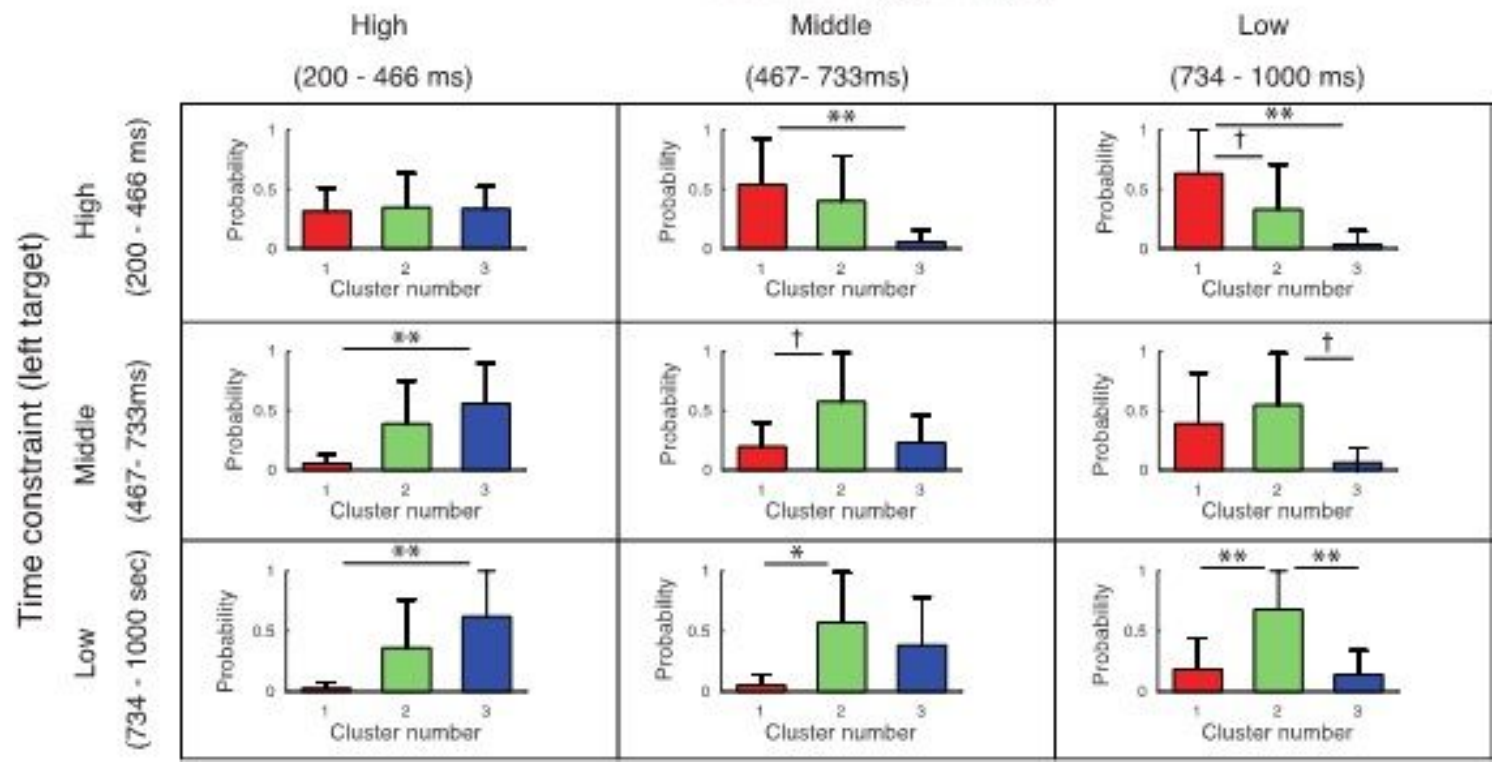

Figure 4

Clustering of the initial movement and the probability of appearance of movement patterns according to time constraints. The upper left panel shows a scatter plot of the movement direction and velocity of the initial movement, including data from all participants. The data were classified into three clusters (red, green, and blue) by K-means clustering for the bivariate initial movement variables (i.e., the IMD and IMV). The upper right panel shows the between-participant mean of the probability of occurrence of each cluster under all time constraints. Error bars are between-participant standard deviations. The bottom panels show the between-participant average of the probability of occurrence of each cluster, depending on the time constraint. This figure shows that when the time constraints are equal, the occurrence probability of the intermediate direction (green) is higher, and with differences in the time constraints, the occurrence probability of the initial movement to the target direction (red and green) with a shorter time constraint is higher. 
Right time-constraint fixed
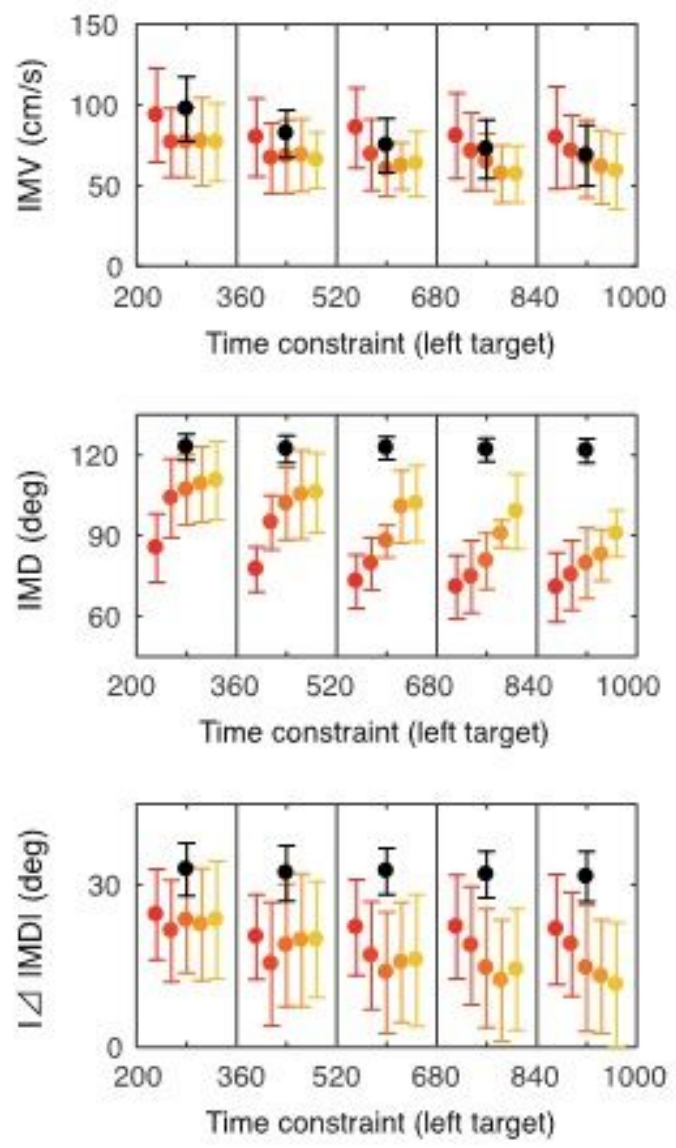

Left time-constraint fixed
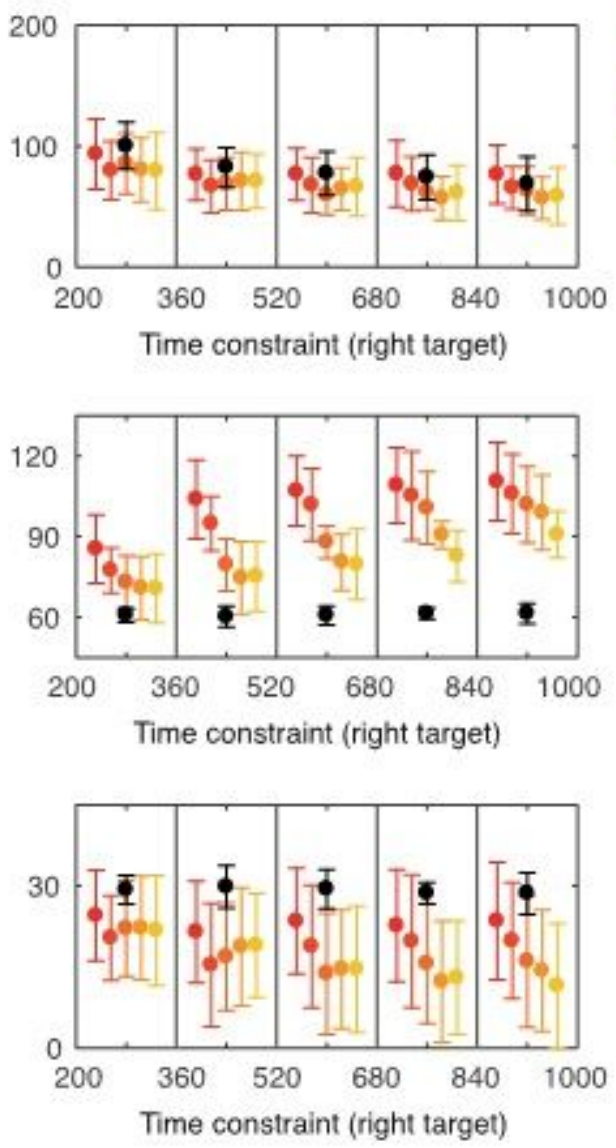

Figure 5

Comparison of the initial movement behavior among conditions. The upper, middle, and lower panels show inter-condition comparisons of the IMD, IMV, and $|\triangle I M D|$, respectively. The circles and error bars show the means and standard deviations, respectively. The black-colored circles show the data for the single-target condition, and gradient circles from red to yellow show the data for the double-target condition. The color changes from red to yellow depending on the severity of the time constraint of the other target (red is tight, yellow is loose). The left and right panels are drawn as a function of the time constraints of the left and right targets, respectively. These panels show the modulation of the initial movement depending on time constraints. 

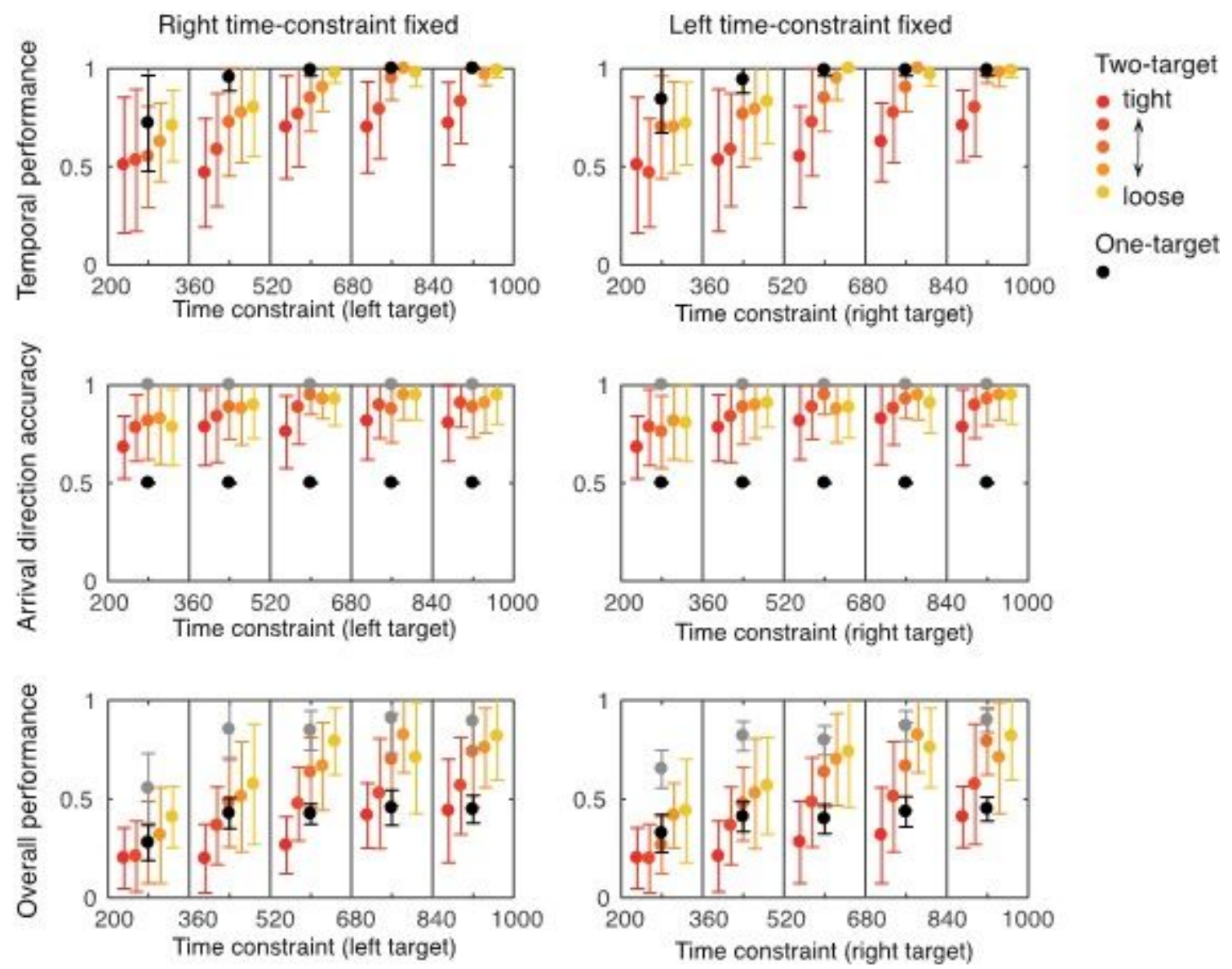

\section{Figure 6}

Comparison of performance (temporal accuracy, arrival target accuracy, overall performance) among conditions. The upper, middle, and lower panels show inter-condition comparisons of the temporal accuracy, arrival target accuracy, and overall performance, respectively. The circles and error bars show the mean and standard deviation, respectively. The gray-colored circles show the data for the single-target condition. The black-colored circles show the estimated performance of predeterminant strategy based on the data in the single-target condition. The gradient circles from red to yellow show the data for the double-target condition. The color changes from red to yellow, depending on the severity of the time constraint of the other target (red is tight, yellow is loose). The left and right panels are drawn as a function of the time constraints of the left and right targets, respectively. The temporal accuracy in the double-target condition is significantly less than that in the single-target condition, and the arrival target accuracy in the double-target condition is significantly higher than the performance of predeterminant strategy estimated from the data of the single-target condition (black-colored circles and lines). Additionally, the overall performance in tight time-constraint condition (red circles and lines in both right and left panels) was significantly less than the performance of the predeterminant strategy (black-colored circles and lines). This deficit in performance may be due to excessive preference for the choice reaction even in the tight time-constraint conditions.

\section{Supplementary Files}


This is a list of supplementary files associated with this preprint. Click to download.

- supplementary210425.docx 